\title{
Investigation of a gradient enriched Gurson-Tvergaard model for porous strain hardening materials
}

Holte, I.; Niordson, C. F. ; Nielsen, K. L.; Tvergaard, V.

Published in:

European Journal of Mechanics A - Solids

Link to article, DOI:

10.1016/j.euromechsol.2019.03.001

Publication date:

2019

Document Version

Peer reviewed version

Link back to DTU Orbit

Citation (APA):

Holte, I., Niordson, C. F., Nielsen, K. L., \& Tvergaard, V. (2019). Investigation of a gradient enriched GursonTvergaard model for porous strain hardening materials. European Journal of Mechanics A - Solids, 75, 472-484. https://doi.org/10.1016/j.euromechsol.2019.03.001

\section{General rights}

Copyright and moral rights for the publications made accessible in the public portal are retained by the authors and/or other copyright owners and it is a condition of accessing publications that users recognise and abide by the legal requirements associated with these rights.

- Users may download and print one copy of any publication from the public portal for the purpose of private study or research.

- You may not further distribute the material or use it for any profit-making activity or commercial gain

- You may freely distribute the URL identifying the publication in the public portal 


\title{
Investigation of a gradient enriched Gurson-Tvergaard model for porous strain hardening materials
}

\author{
I. Holte ${ }^{\mathrm{a}, *}$, C.F. Niordson ${ }^{\mathrm{a}}$, K.L. Nielsen ${ }^{\mathrm{a}}$, V. Tvergaard ${ }^{\mathrm{a}}$ \\ ${ }^{a}$ Department of Mechanical Engineering, Solid Mechanics, Technical University of Denmark, DK-2800 Kgs. Lyngby, \\ Denmark
}

\begin{abstract}
Size effects in a strain hardening porous solid are investigated using the Gurson-Tvergaard (GT) model enriched by a constitutive length parameter, as proposed by Niordson and Tvergaard [C.F. Niordson, V. Tvergaard, A homogenised model for size effects in porous metals, J. Mech. Phys. Solids (2019)]. The results are compared with unit cell calculations of regularly distributed voids embedded in a strain gradient enhanced matrix material. The strain gradient plasticity theory proposed by Fleck and Willis [N.A. Fleck, J.R. Willis, A mathematical basis for strain gradient plasticity theory. Part II: tensorial plastic multiplier, J. Mech. Phys. Solids 57 (2009) 1045-1057], extended to finite strains, is adopted for the cell model, consistent with the gradient enriched Gurson model. The gradient model allows for a material length parameter to enter the constitutive framework for dimensional consistency, while the enriched GT model has the same length parameter introduced through prefactors of the usual $q_{1}$ and $q_{2}$ factors. The continuum model featuring size-dependent Tvergaard-constants is used to investigate a strain hardening material with the strain gradient plasticity enriched cell model as reference. The two models are compared for three triaxialities, three initial void volume fractions, and three hardening exponents. The enriched GT model captures the effect of elevated yield point and suppressed void growth with increasing length parameter for all the cases investigated. The agreement between the models is good until severe void distortion or plastic flow localisation between neighbouring voids. The response curves and void growth curves for the enriched GT model deviate from those of the cell model at high axial strains. Void shape plots, which are only available for the cell model, show that the length parameter influences the shape of the void which in turn has impact on the material response curves and the void evolution. This is not captured by the enriched GT model as the voids are accounted for solely through a volume fraction parameter.
\end{abstract}

Keywords: Size-effects, Voids, Strain-gradient plasticity

\footnotetext{
This article was presented at the IUTAM Symposium on Size-Effects in Microstructure and Damage Evolution at Technical University of Denmark, 2018

*Corresponding author

$U R L$ : inghol@mek.dtu.dk (I. Holte)
} 


\section{Introduction}

Size effects in metal plasticity, exhibiting the general trend that smaller is stronger due to hardening associated with strain gradients, have been confirmed in many experiments. Fleck et al. (1994) showed a size effect in torsion of thin copper wires, with the onset of yielding delayed for diminishing specimen size. Stelmashenko (1993) and Ma and Clarke (1995) have shown that material hardness increases with decreasing indentation size, while Stölken and Evans (1998) documented size effects in bending. Following the work of Asbhy (1970), the apparent flow stress is known to be influenced by both statistically stored dislocations, created during homogeneous strain, and geometrically necessary dislocations which are related to plastic strain gradients. To account for the strain gradient effect, a number of phenomenological theories of plasticity have been developed. One such theory, proposed by Fleck and Hutchinson (1997), includes higher order stresses that are work conjugate to plastic strain gradients. Gudmundson (2004) presented a thermodynamical consistent framework and constitutive laws extending the theory presented by Fleck and Hutchinson (1997). The contribution by Gudmundson (2004), was later reformulated mathematically by Fleck and Willis (2009) and the variational structures of both rate-dependent (visco-plastic) and rate-independent versions was laid out. In the present paper, the visco-plastic formulation of the Fleck-Willis theory is used to perform cell model studies of a discrete void in a gradient enhanced strain hardening matrix. The strain gradient plasticity framework has been implemented numerically by Nielsen and Niordson $(2013,2014)$, and extended to finite strains in a visco-plastic setting by Niordson and Tvergaard (2019).

For porous, ductile media, the voids and their evolution affect material behaviour. In gradient hardening materials, a small void will generate large gradients, and the void evolution will not be correctly captured by classic plasticity theories. Wen et al. (2005) put forth an extension to the Gurson-Tvergaard (GT) model (Tvergaard, 1981; Tvergaard, 1982) by accounting for dislocation hardening based on the theory of plasticity introduced by Gao and Huang (2001). In this theory, Taylor's dislocation hardening model and the density of geometrically necessary dislocations are linked to non-local plasticity. Monchiet and Bonnet (2013) extended the GT model to account for strain gradient effects on cavity growth. Dormieux and Kondo (2010) introduced effects of interface stresses at the cavity surface to the GT model. The interface effect was found to be controlled by a parameter depending on the cavity size, which also affected the macroscopic yield strength of the media. Monchiet and Kondo (2013) extended this to account for non-spherical voids.

Niordson and Tvergaard (2019) investigated size-effects in a porous metal based in cell model analyses of axi-symmetric loading states. They proposed that conventional yield surfaces for porous metals can be extended to account for size-effects by introducing an effective void volume fraction smaller than the actual void volume fraction, and a decreased mean stress sensitivity. The aim of the present paper is to verify the applicability of this proposal to the GT model. The conventional GT model accounts for the presence of voids solely through the the void volume fraction, $f$. To account for size-effects originating from strain gradient effects, Niordson and Tvergaard (2019) proposed to introduce two size dependent parameters, $Q_{1}$ and $Q_{2}$, as prefactors of the void volume fraction and the mean stress, respectively, in the yield condition. A parametric study with this representation of size effects in an extended GT model is presented and the predictions 
are compared with corresponding unit cell model predictions, where a discrete void is embedded in a finite strain gradient matrix material of the Fleck-Willis type.

The paper is structured as follows. The work is outlined in Section 2. The material models are presented in Section 3, the gradient cell model in Section 3.1, and the enriched GT model in Section 3.2. The results are presented and discussed in Section 4, while the work is concluded in Section 5. 


\section{Problem formulation}

Material porosity is modelled using two different approaches. One has voids represented discretely, while the other is based on a homogenised yield function of the Gurson-Tvergaard type.

The discrete model is a unit cell with matrix material governed by the gradient theory by Fleck and Willis (2009) in a finite strain generalisation, and a discretely modelled embedded void (Section 3.1). The cell model approximates an array of voids arranged in a layered hexagonal pattern (see e.g. Niordson and Tvergaard, 2019). Due to symmetry, only half of the cell is modelled with the hexagonal cell approximated by an axi-symmetric unit cell model as shown in Fig. 1b. Fig. 1a shows one cylinder with three planes of voids. The initial void plane distance is $2 H_{c}$, the initial in-plane void distance is $2 R_{c}$, and the initial void radius is denoted $R_{0}$. The initial void volume fraction is thereby given as

$$
f_{0}=\frac{2 R_{0}^{3}}{3 R_{c}^{2} H_{c}} .
$$

The discretely modelled void is initially spherical, but changes shape upon loading, and the shape is characterised by the aspect ratio given by

$$
S=\frac{R_{0}+\Delta_{A}}{R_{0}+\Delta_{B}}
$$

where $\Delta_{A}$ is the displacement in axial direction of the node at the boundary between the discrete void and the matrix aligned with the $x_{1}$-axis, while $\Delta_{B}$ is the displacement in radial direction of the node at the boundary between the discrete void and the matrix aligned with the $x_{2}$-axis (see Fig. 1b). Thus, $S>1$ means the void is prolate, and $S<1$ corresponds to an oblate void. For a spherical void, $S=1$. The numerical analyses are carried out using the finite element method with a mesh consisting of 480 elements, with 24 elements discretising one quarter of the circumference of the void, whereas 20 graded elements are employed in the radial direction. To determine the displacement field, eight-node, isoparametric, axi-symmetric elements are used, whereas for the plastic strain rate field, the corresponding four node elements are used (see also Section 3.1).

The homogenised model is a single point model representing a homogeneous continuum governed by the gradient enriched GT model as proposed by Niordson and Tvergaard (2019), where the porosity is represented by a void volume fraction denoted by $f$ (see Section 3.2). The gradient enriched GT model is solved directly by forward Euler integration of an imposed stress/strain history. The strain gradient plasticity cell model has been used as benchmark for investigation of the enriched GT model in a parametric study. The investigated parameter space is given in Tab. 1.

In both models, a Rayleigh-Ritz method is employed to ensure a constant ratio, $\rho$, of transverse to axial true stresses for each increment of the analyses with a prescribed tensile displacement. The stress triaxiality is related to the stress ratio through

$$
T=\frac{1}{3}\left[\frac{1+2 \rho}{1-\rho}\right] \quad \text { with } \rho=\frac{\sigma_{22}}{\sigma_{11}} .
$$




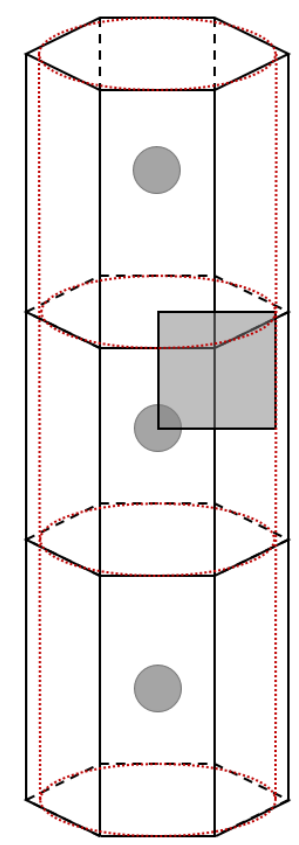

(a)

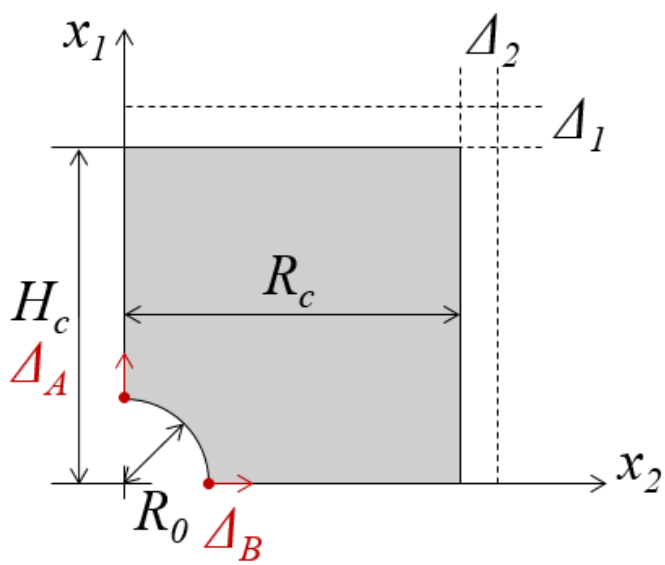

(b)

Fig. 1 a) Voids are assumed periodically arranged in hexagonal cylinders, which are modelled as circular through axi-symmetric boundary conditions. The voids are assumed to be placed in equally spaced planes. The shaded area indicates the unit cell approximation. b) the unit cell model approximation. 
Tab. 1 Values for the parametric study of the gradient enriched GT model. The stress state triaxiality from Eq. (3) is denoted by $T$, while $n$ is the hardening exponent in Eq. (8), $f_{0}$ is the initial void volume fraction in Eq. (1), and $L_{D}$ is the intrinsic length parameter.

\begin{tabular}{|l|l|l|l|}
\hline \multicolumn{2}{|c|}{ Varied parameter } & Fixed parameters & Length scales, $L_{D} / R_{c}$ \\
\hline$T$ & $1,2,3$ & $\begin{array}{l}n=0.1 \\
f_{0}=0.0104\end{array}$ & $0^{a}, 0.05,0.1,0.25,0.5$ \\
\hline$n$ & $0.05,0.1,0.2$ & $\begin{array}{l}T=2 \\
f_{0}=0.0104\end{array}$ & $0,0.05,0.1,0.25,0.5$ \\
\hline$f_{0}$ & $0.0052,0.0104,0.042$ & $\begin{array}{l}T=2 \\
n=0.1\end{array}$ & $0,0.05,0.1,0.25,0.5$ \\
\hline
\end{tabular}

${ }^{a}$ The simulation has been carried out with an intrinsic length scale of $\frac{L_{D}}{R_{c}}=10^{-4}$, which is considered sufficiently small for the material to be referred to as a conventional material. 


\section{Material models}

\subsection{Strain gradient plasticity model}

The strain gradient plasticity model is based on the visco-plastic strain gradient plasticity theory proposed by Gudmundson (2004) in the context of the mathematical formulation in terms of minimum principles as proposed by Fleck and Willis (2009). Here, the model presentation is kept brief and the reader is referred to Niordson and Tvergaard (2019) and Nielsen and Niordson (2018) for details on the finite strain extension. The theory accounts for internal elastic energy storage due to elastic strain and for dissipation due to the plastic strain rate, $\dot{\varepsilon}_{i j}^{p}$ and its spatial gradient, $\dot{\varepsilon}_{i j, k}^{p}$. The principle of Virtual Work (PVW) in Cartesian components is expressed by

$$
\int_{V}\left(\sigma_{i j} \delta \dot{\varepsilon}_{i j}+\left(q_{i j}-s_{i j}\right) \delta \dot{\varepsilon}_{i j}^{p}+\tau_{i j k} \delta \dot{\varepsilon}_{i j, k}^{p}\right) \mathrm{d} V=\int_{S}\left(T_{i} \delta \dot{u}_{i}+t_{i j} \delta \dot{\varepsilon}_{i j}^{p}\right) \mathrm{d} S
$$

where $\sigma_{i j}$ and $s_{i j}=\sigma_{i j}-\frac{1}{3} \delta_{i j} \sigma_{k k}$ are the Cauchy stress tensor and the stress deviator, respectively. The micro-stress, $q_{i j}$, is work conjugate to the plastic strain rate, $\dot{\varepsilon}_{i j}^{p}$, and $\tau_{i j k}$ is a higher order stress, work conjugate to the plastic strain rate gradient, $\dot{\varepsilon}_{i j, k}^{p}$. The outward unit normal to the surface $S$ is $n_{i}$. The right hand side of the PVW includes the conventional traction, $T_{i}=\sigma_{i j} n_{j}$ work conjugate to the boundary displacement rate, $\dot{u}_{i}$, and the higher order traction, $t_{i j}=\tau_{i j k} n_{k}$, work conjugate to the plastic strain rate, $\dot{\varepsilon}_{i j}^{P}$. Balance laws for the stress quantities follow directly:

$$
\begin{gathered}
\sigma_{i j, j}=0 \\
q_{i j}-s_{i j}-\tau_{i j k, k}=0
\end{gathered}
$$

where, the first set of equations are the conventional equilibrium equations in the absence of body forces, and the second set are the higher order equilibrium equations.

\subsubsection{Constitutive equations}

The rate-dependent visco-plastic formulation employs a visco-plastic potential to account for plastic dissipation as follows

$$
\Phi\left[\dot{E}^{P}, E^{P}\right]=\int_{0}^{\dot{E}^{P}} \sigma_{c}\left[\dot{E}^{P^{\prime}}, E^{P}\right] \mathrm{d} \dot{E}^{P^{\prime}}
$$

Here, $\sigma_{c}$ is the gradient enhanced effective stress, related to the current matrix flow stress through $\sigma_{c}=\sigma_{F}\left[E^{p}\right]\left(\frac{\dot{E}^{p}}{\dot{\varepsilon}_{0}}\right)^{m}$, with $\dot{\varepsilon}_{0}$ denoting the reference strain rate, and $m$ denoting the rate-sensitivity exponent. For the strain hardening material in this paper, the matrix flow stress is given by the isotropic power law

$$
\sigma_{F}=\sigma_{y}\left(1+\frac{E^{p}}{\sigma_{y} / E}\right)^{n}
$$

where $\sigma_{y}$ is the initial matrix material yield stress. A gradient enhanced effective plastic strain rate is introduced by 


$$
\left(\dot{E}^{p}\right)^{2}=\frac{2}{3} \dot{\varepsilon}_{i j}^{p} \dot{\varepsilon}_{i j}^{p}+L_{D}^{2} \dot{\varepsilon}_{i j, k}^{p} \dot{\varepsilon}_{i j, k}^{p}
$$

and the associated work conjugate gradient enhanced effective stress is given by

$$
\sigma_{c}^{2}=\frac{3}{2} q_{i j} q_{i j}+\frac{1}{L_{D}^{2}} \tau_{i j k} \tau_{i j k}
$$

where $L_{D}$ is a dissipative constitutive length parameter that enters for dimensional consistency. The dissipative stress quantities are given by

$$
q_{i j}^{D}=\frac{2}{3} \sigma_{c} \frac{\dot{\varepsilon}_{i j}^{p}}{\dot{E}^{p}}, \quad \tau_{i j k}^{D}=L_{D}^{2} \sigma_{c} \frac{\dot{\varepsilon}_{i j, k}^{p}}{\dot{E}^{p}} .
$$

The superscript $D$ refers to dissipative quantities.

\subsubsection{Solution method}

The incremental boundary value problem is solved using the Finite Element Method based on the two minimum principles set forward by Fleck and Willis (2009) (see Niordson and Tvergaard (2019) for details on the finite strain formulation). A forward Euler integration scheme is employed throughout Minimum Principle I, which for the time dependent solutions includes the visco-plastic potential, and reads

$$
H=\inf _{\dot{\varepsilon}_{i j}^{p}} \int_{V}\left(\Phi\left[E^{p}, \dot{E}^{p}\right]+\tau_{i j k}^{E} \dot{\varepsilon}_{i j, k}^{p}-s_{i j} \dot{\varepsilon}_{i j}^{p}\right) \mathrm{d} V-\int_{S} t_{i j} \dot{\varepsilon}_{i j}^{p} \mathrm{~d} S
$$

The superscripts $E$ refers to the energetic contributions. Assuming that the current state of stress, $\sigma_{i j}$, and the plastic deformation, $\varepsilon_{i j}^{P}$, are known everywhere in the volume, the plastic strain rate field, $\dot{\varepsilon}_{i j}^{p}$, for each time step is found by requiring Eq. (12) stationary through

$$
\int_{V}\left(q_{i j}^{D} \delta \dot{\varepsilon}_{i j}^{p}+\tau_{i j k}^{D} \delta \dot{\varepsilon}_{i j, k}^{p}\right) \mathrm{d} V=\int_{V}\left(s_{i j} \delta \dot{\varepsilon}_{i j}^{p}-\tau_{i j k}^{E} \delta \dot{\varepsilon}_{i j, k}^{p}\right) \mathrm{d} V+\int_{S} t_{i j} \delta \dot{\varepsilon}_{i j}^{p} \mathrm{~d} S
$$

Equation (13) is solved by an iterative procedure. This problem is taken to be purely dissipative and $\tau_{i j k}^{E}$ is zero. The dissipative stress quantities, $q_{i j}^{D}$ and $\tau_{i j k}^{D}$ are found from Eq. (11). Once the plastic strain rate field is determined, Minimum Principle II (Eq. (14))

$$
J\left[\dot{u}_{i}\right]=\frac{1}{2} \int_{V} L_{i j k l}\left(\dot{\varepsilon}_{i j}-\dot{\varepsilon}_{i j}^{p}\right)\left(\dot{\varepsilon}_{k l}-\dot{\varepsilon}_{k l}^{p}\right) \mathrm{d} V-\int_{S} \dot{T}_{i} \dot{u}_{i} \mathrm{~d} S .
$$

is used to calculate the corresponding displacement field in an updated Lagrangian setting following McMeeking and Rice (1975). The incrementation of the Fleck and Willis theory is conducted using a forward Euler integration scheme built into an in-house FORTRAN code. For details on the finite strain implementation, the reader is referred to Niordson and Tvergaard (2019) and Nielsen and Niordson (2018). 


\subsection{The gradient enriched Gurson model}

The basis for the gradient enriched continuum model is a finite-strain formulation of the GursonTvergaard model, which describes the behaviour of a porous elastic-plastic solid as dilating, pressure sensitive plastic flow of a solid with the yield condition $\Phi\left(\sigma_{i j}, \sigma_{M}, f\right)=0$ (see Gurson, 1977; Tvergaard, 1982). Here, $\sigma_{i j}$ is the average macroscopic Cauchy stress tensor, $\sigma_{M}$ is the equivalent tensile flow stress of the matrix material, and $f$ is the current void volume fraction. The Gurson model used here is time-independent while the gradient model is visco-plastic. Comparison is reasonable as the rate sensitivity exponent is taken to be very small. Niordson and Tvergaard (2019) recently presented a simple method for transforming a conventional yield surface for a porous material so that it accounts for size-effects. In the context of the GT model their proposal is to introduce two size-dependent parameters, $Q_{1}$ and $Q_{2}$, as prefactors to the conventional Tvergaard parameters $q_{1}$ and $q_{2}$, so that the yield condition reads:

$$
\Phi=\frac{\sigma_{e}^{2}}{\sigma_{M}^{2}}+2 Q_{1} q_{1} f \cosh \left\{\frac{Q_{2} q_{2}}{2} \frac{\sigma_{k k}}{\sigma_{M}}\right\}-\left[1+\left(Q_{1} q_{1} f\right)^{2}\right]=0
$$

where $\sigma_{e}=\left(3 s_{i j} s_{i j} / 2\right)^{1 / 2}$ is the macroscopic equivalent stress, $s_{i j}=\sigma_{i j}-\frac{1}{3} \delta_{i j} \sigma_{k k}$ is the macroscopic Cauchy stress deviator, and $\delta_{i j}$ is the Kronecker delta. The gradient enriched $Q_{1}$ and $Q_{2}$ factors are given by:

$$
\begin{gathered}
Q_{1} \approx \frac{0.364}{1+1.8\left(\frac{L_{D}}{R_{V}}\right)+10\left(\frac{L_{D}}{R_{V}}\right)^{2}}+0.636, \\
Q_{2} \approx \frac{1}{1+1.8\left(\frac{L_{D}}{R_{V}}\right)^{3 / 2}}
\end{gathered}
$$

In order to capture the evolution of size-effects with void volume fraction, the ratio of the material length scale to void size is taken to develop with the cubic root of the inverse void volume fraction as follows:

$$
\frac{L_{D}}{R_{V}}=\frac{L_{D}}{R_{0}}\left(\frac{f_{0}}{f}\right)^{1 / 3}
$$

Here, the intrinsic length parameter is $L_{D}, R_{0}$ is the initial void radius corresponding to the initial void volume fraction, $f_{0}$, and $R_{v}$ is the current void radius corresponding to a current void volume fraction, $f$, assuming spherical voids. Eq. (18) shows how the current void radius, $R_{V}$, relates to the void growth. It should be noted that for $L_{D}=0$, the prefactors $\left(Q_{1}\right.$ and $\left.Q_{2}\right)$ become one and the yield condition reduces to that of Gurson (1977) modified by Tvergaard $(1981,1982)$ through the Tvergaard-constants, here taken to be $q_{1}=1.5$ and $q_{2}=1$.

The incremental relationship between the microscopic equivalent plastic strain and the microscopic equivalent stress is $h_{M}=\mathrm{d} \sigma_{M} / \mathrm{d} \varepsilon_{M}^{P}$, with microscopic equivalent plastic strain, $\varepsilon_{M}^{P}$, in the matrix varying according to the equivalent plastic work expression

$$
\sigma_{i j} \dot{\varepsilon}_{i j}^{P}=(1-f) \sigma_{M} \dot{\varepsilon}_{M}^{P}
$$


Combining the plastic work expression and the incremental relationship between microscopic stress and strain, gives the following expression for $\dot{\sigma}_{M}$

$$
\dot{\sigma}_{M}=h_{M} \frac{\sigma_{i j} \dot{\varepsilon}_{i j}^{P}}{(1-f) \sigma_{M}} .
$$

The matrix material satisfies the plastic incompressibility condition. However, the presence and growth of voids are associated with volume changes, thus the trace of the plastic deformation rate becomes nonzero. In this work, neither void nucleation nor coalescence is considered and the porosity growth rate is taken only to be dependent on the plastic deformation rate through

$$
\dot{f}=(1-f) \dot{\varepsilon}_{k k}^{P} .
$$

Following Bishop and Hill (1951) and Gurson (1977) normality locally within the matrix implies macroscopic normality. Thus, the plastic strain rate tensor must be normal to the yield surface according to

$$
\dot{\varepsilon}_{i j}^{P}=\Lambda \frac{\partial \Phi}{\partial \sigma_{i j}}
$$

The plastic multiplier, $\Lambda$, is determined by substituting Eqs. (20) and (21) into the consistency condition during plastic straining. The current void radius is a function of $f$, hence evolution of the parameters $Q_{1}$ and $Q_{2}$ must be accounted for in the consistency condition, which then reads $\dot{\Phi}\left(\sigma_{i j}, \sigma_{M}, f, Q_{1}, Q_{2}\right)=0$, with $\dot{Q}_{1}$ and $\dot{Q}_{2}$ being functions of $f$. By solving for $\Lambda$, Eq. (22) can be written as

$$
\dot{\varepsilon}_{i j}^{P}=\frac{1}{H} n_{i j} n_{k l} \dot{\sigma}_{k l}, \quad \text { with } \quad n_{i j}=\frac{3}{2} \frac{s_{i j}}{\sigma_{M}}+\alpha \delta_{i j},
$$

where $H$ is given by

$$
\begin{gathered}
H=\frac{h_{M}}{(1-f)}\left(\omega+\alpha \frac{\sigma_{k k}}{\sigma_{M}}\right)^{2}-3 \sigma_{M}(1-f) \alpha\left[\gamma\left(1+\frac{f}{Q_{1} q_{1}} \frac{\partial Q_{1}}{\partial f}\right)+\frac{\alpha}{Q_{2} q_{2}} \frac{\sigma_{k k}}{\sigma_{M}} \frac{\partial Q_{2}}{\partial f}\right] \\
\alpha=\frac{1}{2} Q_{1} q_{1} Q_{2} q_{2} f \sinh \left(\frac{Q_{2} q_{2}}{2} \frac{\sigma_{k k}}{\sigma_{M}}\right) \\
\gamma=Q_{1} q_{1} \cosh \left(\frac{Q_{2} q_{2}}{2} \frac{\sigma_{k k}}{\sigma_{M}}\right)-\left(Q_{1} q_{1}\right)^{2} f \\
\omega=\frac{\sigma_{e}^{2}}{\sigma_{M}^{2}}
\end{gathered}
$$

The total strain increment is given by $\dot{\varepsilon}_{i j}=\dot{\varepsilon}_{i j}^{E}+\dot{\varepsilon}_{i j}^{P}$, with the elastic rate of deformation taken to be

$$
\dot{\varepsilon}_{i j}^{E}=\frac{1+v}{E} \stackrel{\nabla}{\sigma}_{i j}-\frac{v}{E} \delta_{i j} \stackrel{\nabla}{\sigma}_{k k}
$$

with, $\stackrel{\nabla}{\sigma}_{i j}$ denoting the Jaumann stress rate. For the single-point model, there is no distinction between the deformed and reference configuration which allows for the rigid body rotations to 
be omitted. The spin tensor is zero and the incremental Cauchy stress may therefore be used directly. Adding the elastic and plastic rate of deformation, and inverting, gives the following relation between the stress and strain increment

$$
\dot{\sigma}_{i j}=\mathbb{L}_{i j k l} \dot{\varepsilon}_{i j}
$$

with $\mathbb{L}_{i j k l}$

$$
\mathbb{L}_{i j k l}=\mathcal{L}_{i j k l}-\mu M_{i j} M_{k l}
$$

where $\mathcal{L}_{i j k l}$ is the elastic stiffness tensor. $M_{i j}$ and $\mu$ are given by

$$
M_{i j}=\mathcal{L}_{i j k l} n_{k l}, \quad \mu=\frac{1}{H+\mathcal{L}_{i j k l} n_{i j} n_{k l}} .
$$

It is important to note that the parameters $Q_{1}$ and $Q_{2}$ enter the hardening modulus in Eq. (24), and complicates the implementation of the enriched GT model. However, it will be discussed in Section 4.4 that the effect of omitting the variation of $Q_{1}$ and $Q_{2}$ with $f$ is minor. The enriched GT model is solved with a forward Euler integration scheme. 


\section{Numerical results and discussion}

Solutions based on the gradient enriched GT model are presented through a parametric study and compared to corresponding predictions from the unit cell model. Throughout the parameters $\sigma_{y} / E=0.005, v=0.3$ and $m=0.01$ are used. The strain rate sensitivity parameter, $m$, enters the cell model only, and care has been taken that visco-plastic effects are very limited (see also [20]). The enriched GT model is run with a step size of $\varepsilon_{0} / 100$, where $\varepsilon_{0}=\sigma_{y} / E$, to match the results from the Fleck and Willis governed cell model. The loading rate in the cell model is equal to the reference strain, $\varepsilon_{0}$. The influence of the stress state triaxiality, $T$, the matrix material strain hardening, $n$, and the initial void volume fraction, $f_{0}$ will be studied in this section. Finally, the effect of neglecting the effect of the derivatives of $Q_{1}$ and $Q_{2}$ in the consistency condition will be discussed.

\subsection{Effect of triaxiality}

To investigate the effect of stress triaxiality, the hardening exponent is kept constant at $n=0.1$ and the initial void volume fraction at $f_{0}=0.0104$. The triaxialities investigated are $T=1,2$ and 3 , which corresponds to $\rho$-values of $0.4,0.625$ and 0.727 (see Eq. (3)). The response curves and void growth curves for a conventional material are presented alongside results from simulations of materials with four different length parameters: $\frac{L_{D}}{R_{c}}=0.05,0.1,0.25$ and 0.5 in Fig. 2 . The conventional material is modelled with an intrinsic length parameter approaching zero. A small intrinsic length parameter corresponds to a microstructure where plastic gradient effects play little part such that the effective plastic strain in Eq. (9) equals the von Mises equivalent strain. In contrast, a larger intrinsic length parameter gives rise to greater gradient contributions. It should be noted that increasing the intrinsic length parameter corresponds to a material with smaller voids, but of the same initial void volume fraction (void are smaller and located closer together). The response curves show the true axial stress as a function of logarithmic axial strain, while the void growth curves show the relative void volume fraction as a function of logarithmic axial strain. It is seen that the gradient enriched GT model captures the increased gradient hardening reflected in all response curves, as well as predicts suppressed void growth with increasing length parameter seen for all void growth curves. For the lowest stress triaxiality considered $(T=1)$, the results from the enriched Gurson model fit well with the results from the cell model and only a small variation in gradient hardening is predicted. At this stress state, the stresses in axial direction are sufficiently large compared to those in the radial direction to develop localisation in the intervoid ligaments, and the material will experience only strain and gradient hardening. Little void growth occurs for these simulations, and the voids are merely stretched along the main straining axis for the values of logarithmic axial strain presented here. The void shape evolution curves from the cell model simulations presented in Fig. 3 show the shape parameter, $S$, plotted against logarithmic axial strain (see Eq. (2)). Recall that for $S>1$ the void is prolate and for $S<1$ the void is oblate. Fig. 3a shows that the voids grow into a prolate shape for all the materials simulated under $T=1$ loading conditions. At the state of deformation considered, the unit cell is not stretched sufficiently for localisation and material softening to occur.

For the higher triaxiality, $T=2$, a greater effect of the gradients is predicted. The enriched Gurson model captures the yield point for all unit cell simulations. Moreover, materials with 
large intrinsic length parameters $\left(\frac{L_{D}}{R_{c}}=0.25\right.$ and 0.5$)$, matches both the response curves and the void growth curves. For such large length parameters, the material does not soften nor does it experience significant void growth. The void shape curves in Fig. $3 \mathrm{~b}$ prove that the voids grow to a prolate shape and the material behaviour resembles that of a conventional material under low stress triaxiality loading conditions. For the conventional material subject to $T=2$, however, both material softening and extensive void growth occurs, and the gradient enriched GT model deviates from the cell model results at high axial strains. The void shape curve for $\frac{L_{D}}{R_{c}} \rightarrow 0$ in Fig. $3 \mathrm{~b}$ shows that the void rapidly grows oblate at the axial strain where the material begins to soften. The oblate shape indicates that the void growth occurs in the radial direction and the voids deform towards coalescence. The enriched GT model will not capture this as it incorporates neither void shape changes nor a localisation criterion.

The response curves and void growth curves for the highest triaxiality, $T=3$, are a continuation of the development found for $T=2$. The conventional material undergoes localisation rapidly and the enriched GT model results deviate from those of the cell model. The materials with the two highest intrinsic length parameters $\left(\frac{L_{D}}{R_{c}}=0.25\right.$ and 0.5$)$ also here exhibit the same behaviour as materials subject to low stress triaxiality loading, and neither significant void growth nor material softening will initiate at the deformation state considered. Fig. 3c shows that voids in a material with insufficient gradient hardening subject to a high stress triaxiality will grow oblate and soften the material through void coalescence.

Deformed meshes and contour plots of the gradient enhanced effective plastic strain, $E^{p}$, is shown in Fig. 4 as obtained by the cell model analyses. The results illustrate the limitations of the gradient enriched Gurson model. Fig. 4 presents results for both the conventional material and the gradient hardening material with intrinsic length parameter $\frac{L_{D}}{R_{c}}=0.1$ for the three different stress triaxialities. It is seen that the voids are more prone to grow oblate at high triaxiality, which is in agreement with results from Koplik and Needleman (1988). Budiansky et al. (1982) studied void shape evolution for a linearly viscous material and found that the void equator lengthens more rapidly than the meridians for a void in a high triaxiality stress field. Gradient hardening, however, affects the void growth for micron scale voids and hence the void shape is affected. Fig. 4 shows the difference in void evolution for the conventional material and the material with intrinsic length parameter $\frac{L_{D}}{R_{c}}=0.1$. The conventional material does not experience gradient hardening and significant void growth takes place. For the material with intrinsic length parameter $\frac{L_{D}}{R_{c}}=0.1$, gradient hardening is sufficient to restrict void growth almost entirely. The void is stretched along the main straining axis and shortened along the equator, growing into a prolate shape. The unit cell is strained accordingly and the relative void volume fraction will therefore not change significantly. For $T=3$, the void in the conventional material matrix grows to an oblate shape, while the void in the gradient hardening in the material with an intrinsic length parameter $\frac{L_{D}}{R_{c}}=0.1$, becomes prolate.

\subsection{Effect of the strain hardening exponent}

Results for three values of the strain hardening exponent, $n$, are presented in the following: $n=0.05$, 0.1 and 0.2 . The stress triaxiality is kept constant at $T=2$ and the initial void volume fraction con- 
stant at $f_{0}=0.0104$. The results for the conventional material, $\frac{L_{D}}{R_{c}} \rightarrow 0$, are presented alongside results from the same four length parameters as for the investigation of stress triaxiality presented in Section 4.1. The response curves and void growth curves are given in Fig. 5, while the void shape curves are presented in Fig. 6. The same quantities as for the triaxiality results in Fig. 2 are plotted for consistency. The strain hardening exponent largely influences the peak of the response curves, and the results for the different values of $n$ can therefore not be compared directly. The simulations for $n=0.2$ are therefore taken to twice as large axial strain compared to the other simulations. The response curves in Fig. 5 show that the enriched Gurson model captures the yield point for all configurations of strain hardening and gradient hardening. The trend of a rising response curve with increasing hardening exponent is also captured. For $n=0.05$, the strain hardening is small and the response curves only show little hardening. The spread in the curves is due to gradient hardening, which is seen to have a small effect for this low value of $n$. For materials with large intrinsic length parameters $\left(\frac{L_{D}}{R_{c}}=0.25\right.$ and 0.5$)$ softening is not observed at the state of deformation considered. The void growth curves show that the relative void volume fraction is nearly constant throughout the simulations, while the void shape curves show that the voids become prolate (see Fig. 6a). The gradient effects are the same here as for the results from the triaxiality study. The response curve for the conventional material with $n=0.05$ follow that of the cell model well until an abrupt change in load carrying capacity of the materials is observed. The logarithmic axial strain at localisation, i.e. the abrupt change in load bearing capacity, corresponds to the strain at which the void shape rapidly grows oblate in Fig. 6 a.

Materials with the highest hardening exponent, $n=0.2$, shows the greatest effect of the gradient hardening. The response curves and void growth curves have a larger spread when compared to those from simulations with $n=0.05$ and 0.1 . This indicates the synergy effect between strain hardening and gradient hardening related to Eq. (9). The materials that do not undergo sufficient gradient hardening to impede the void growth experience extensive hardening before a sudden and rapid material softening. This is not captured well by the enriched GT model, which suggest that the effect of gradient hardening on the void shape evolution is prominent. The void shape curves in Fig. 6c provide further insight on this. It is seen that voids in all the materials with $n=0.2$ become prolate at low axial strains which indicates that the hardening in the material matrix is sufficient to delay excessive void growth in the radial direction. The conventional material experiences the least amount of hardening at lower deformation levels as compared to larger values of $\frac{L_{D}}{R_{c}}$. The other materials show increased hardening with increasing length parameter. High stresses are required to maintain plastic flow in a material with a large hardening exponent, and the localisation will be dramatic making the subsequent softening abrupt. The matrix material is carrying high stresses that will be released at the onset of localisation and the void will grow in the radial direction changing the shape. When comparing Fig. 5 for $n=0.2$ and Fig. $6 \mathrm{c}$ for the conventional material and the material with the lowest length parameter, is it seen that the curves peak at the same value of logarithmic axial strain. Representing the effect of the voids through a single parameter, namely the void volume fraction, $f$, is not sufficient to capture these combined effects, and the enriched GT model will therefore not accurately represent both the response and void growth curves of the cell model. The materials with larger intrinsic length parameter and 
thereby increased gradient hardening will not undergo extensive void growth nor localisation and the gradient enriched Gurson model therefore captures both the response curves and the void growth curves well.

The effect of gradient hardening on the void evolution can be seen in Fig. 7 for the highest value of strain hardening. Deformed meshes and contour plots of $E^{p}$ are depicted for both the conventional material and the materials with smallest and largest intrinsic material length parameters $\left(\frac{L_{D}}{R_{c}}=0.05\right.$ and 0.5$)$ are presented for three different values of logarithmic axial strain. For the conventional material, localisation is seen to have initiated at an intermediate axial strain value, while severe deformation is predicted in the transverse void ligament at the highest value of axial strain. For the material with the lowest intrinsic length parameter, $\frac{L_{D}}{R_{c}}=0.05$, the void is seen to develop in a more restrained manner. At the intermediate axial strain, the void has grown, but still has a prolate shape. For the largest axial strain, the void has returned to a spherical shape, indicating that the void growth occurs mainly in the transverse direction and that localisation has initiated. The material with the highest amount of gradient hardening exhibits only little void growth, but also stretching in conjunction with the unit cell itself.

\subsection{Effect of the initial void volume fraction}

Keeping the triaxiality constant at $T=2$ and the hardening exponent at $n=0.1$, the effect of the initial void volume fraction is investigated. Three initial porosities are considered: $f_{0}=0.0052$

0.0104 and 0.042 . For a square unit cell, given in Fig. (1b) with $\frac{H_{c}}{R_{c}}=1$, this corresponds to initial void radii: $R_{v}=0.2,0.25$ and 0.4 , respectively. The response curves and void growth curves for the three $f_{0}$-values for a conventional material are presented in Fig. 8 together with four materials with length parameters. The void shape evolution is presented in Figure 9.

The response curves in Fig. 8 show that increasing $f_{0}$ decreases the yield point, which corresponds well with results from Niordson and Tvergaard (2019). For the two materials with most gradient hardening, the material does not soften for either value of $f_{0}$ at the state of deformation considered. The void growth curves show that the relative void volume fraction is essentially constant through the simulation for the materials with intrinsic length parameters $\frac{L_{D}}{R_{c}}=0.25$ and 0.5. This indicates that the effect of gradient hardening surpasses the effect of $f_{0}$. However, $f_{0}$ has an effect on both the response curves and the void growth curves for all materials considered independent of length parameter. For an initial void volume fraction of 0.0052 , the conventional material undergoes localisation which is not captured by the enriched Gurson model. The void shape curve for this material shows that the void grows oblate at an accelerated speed at the value of axial strain corresponding to localisation in Fig. 8. Increasing $f_{0}$, gives a larger spread in the response curve yield point indicating that for large voids, gradient hardening has a more prominent effect on the material behaviour than softening.

The material response curves for the largest initial void volume fraction, $f_{0}=0.042$, exhibits a great dependence on the intrinsic length parameter. The conventional material along with the material with the smallest intrinsic length parameter, $\frac{L_{D}}{R_{c}}=0.05$, both soften extensively. It is seen that the voids in the conventional material and the material with the smallest intrinsic length parameter grow to an oblate shape. The connection between oblate voids and material softening corresponds 
well with the results presented for both the investigation of triaxiality and the strain hardening exponent. It is discovered that increasing the initial void volume fraction leads to suppressed relative void growth rate. This seems to be contradictory to previous results, where material softening was an effect of an increasing void volume fraction due to void growth. Deformed meshes and the contours of $E^{p}$ are presented in Figure 10 for all three values of $f_{0}$ with $\frac{L_{D}}{R_{c}}=0.05$ at two values of logarithmic axial strain. These meshes provide an explanation for the suppressed void growth with increasing initial void volume fraction. Although the growth for $f_{0}=0.042$ seems to be considerably more than for the other two values of $f_{0}$, the relative void evolution is not. The deformed meshes in Fig. 10 are from the material with the lowest intrinsic length parameter, which only undergoes localisation for the largest initial void volume fraction. The size of the discrete void makes the transverse ligament too small to sustain the loads. The plastic flow will localise, as shown in Figure 10, while the void is stretched in conjunction with the unit cell, making the void volume fraction nearly constant while the material softens. Increasing the gradient hardening will suppress this effect such that the material will not undergo localisation.

\subsection{Approximation of the consistency condition for the enriched Gurson model}

For all previous figures, the full effect of Eq. (24) has been accounted for. The effect of neglecting $\dot{Q}_{1}$ and $\dot{Q}_{2}$ is investigated in the following by running simulations with an approximation of the consistency condition where $\dot{Q}_{1}$ and $\dot{Q}_{2}$ are omitted. The results are presented in Fig. 11 for simulations with $T=2, n=0.1$ and $f_{0}=0.0104$. The increment size is $\varepsilon_{0} / 100$, where $\varepsilon_{0}=\sigma_{y} / E$. As must be expected, an increasing discrepancy between results for increasing length parameters is seen. Essentially, the hardening modulus, $H$, from Eq. (24), exhibits the largest difference with and without accounting for $\dot{Q}_{1}$ and $\dot{Q}_{2}$ when $L_{D}$ increases until the length parameter becomes large enough for the results to fall on top of each other. For $\frac{L_{D}}{R_{c}} \rightarrow 0$, the results will coincide as the gradient effects are small and the material approaches that of the conventional Gurson-Tvergaard model, which does not incorporate a length parameter. $Q_{1}$ and $Q_{2}$ will approach unity and the response curves in Fig. 11 will fall on top of each other for materials with length parameters approaching zero. For large length parameters, i.e. small void sizes, both $Q_{1}$ and $Q_{2}$ will approach the lower bound value, and $\dot{Q}_{1}$ and $\dot{Q}_{2}$ will approach zero, according to the analyses by Niordson and Tvergaard (2019). For $\frac{L_{D}}{R_{c}}=0.25$ and above, $\dot{Q}_{1}$ and $\dot{Q}_{2}$ have been found to have a negligible effect on the material response.

We conclude that all though the rigorous inclusion of the derivatives of $Q_{1}$ and $Q_{2}$ in the yield condition is needed for a self-consistent framework, the simpler framework where they are neglected provides a reasonable approximation, that could potentially be simpler to implement in existing computational frameworks for porous metal plasticity. 

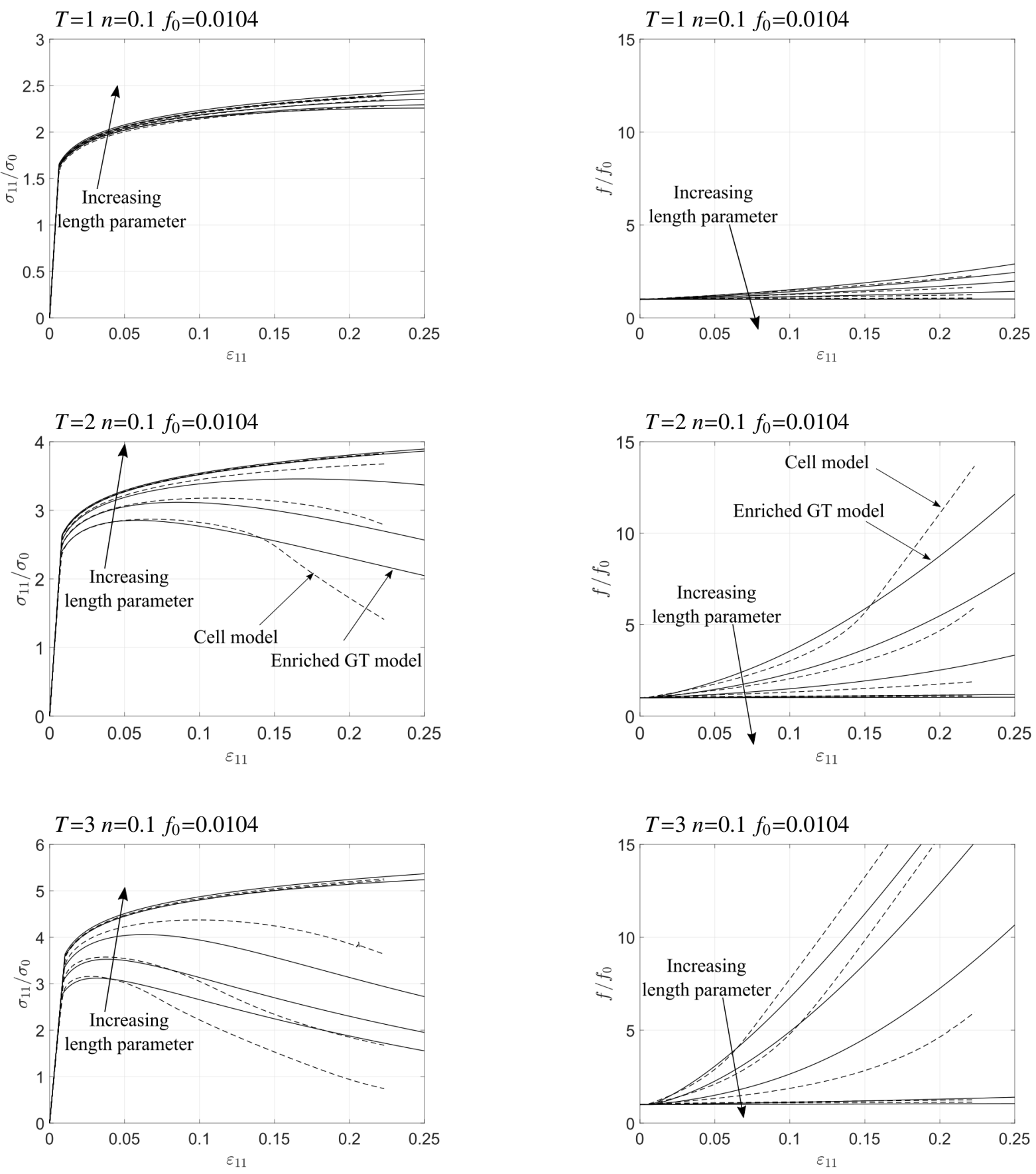

Fig. 2 Finite element results for $f_{0}=0.0104$ and $n=0.1$ with stress triaxialities $T=1,2$ and 3 (from the top down.) The left column shows the macroscopic true axial stress-logarithmic axial strain response. The logarithmic strain is given as $\varepsilon_{11}=\ln \left(1+e_{11}\right)$, where $e_{11}$ is the engineering strain. The right column shows the void volume fraction as function of logarithmic axial strain. The solid lines are the results for the enriched Gurson model, and the cell model results are displayed through the dashed lines. The five length parameters are $\frac{L_{D}}{R_{c}}=0,0.05,0.1,0.25$ and 0.5 . 
(a)

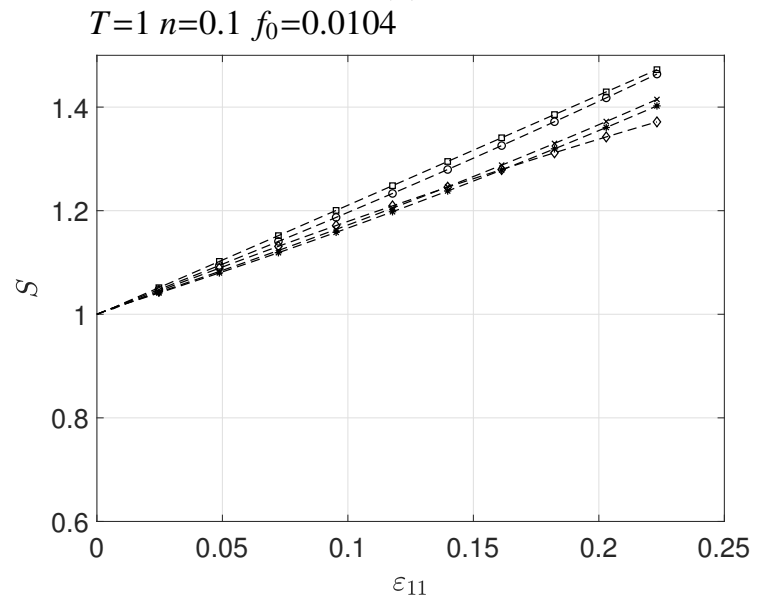

(b)

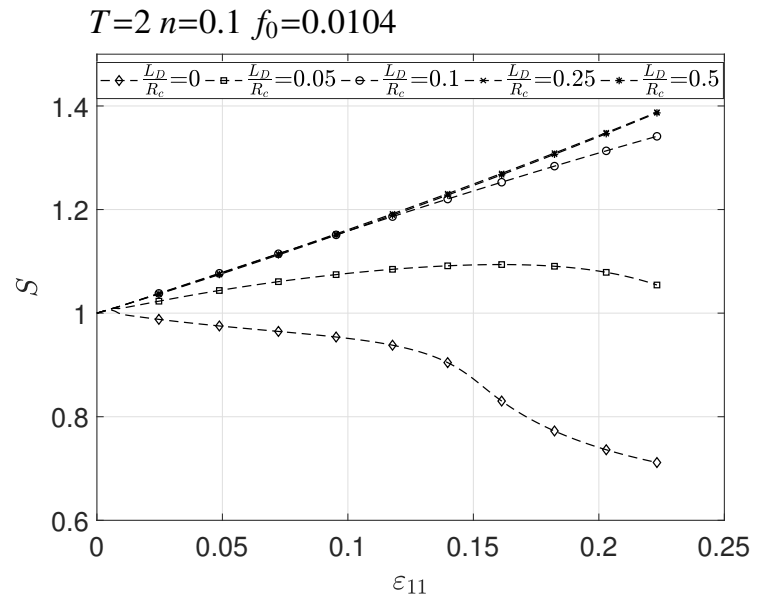

(c)

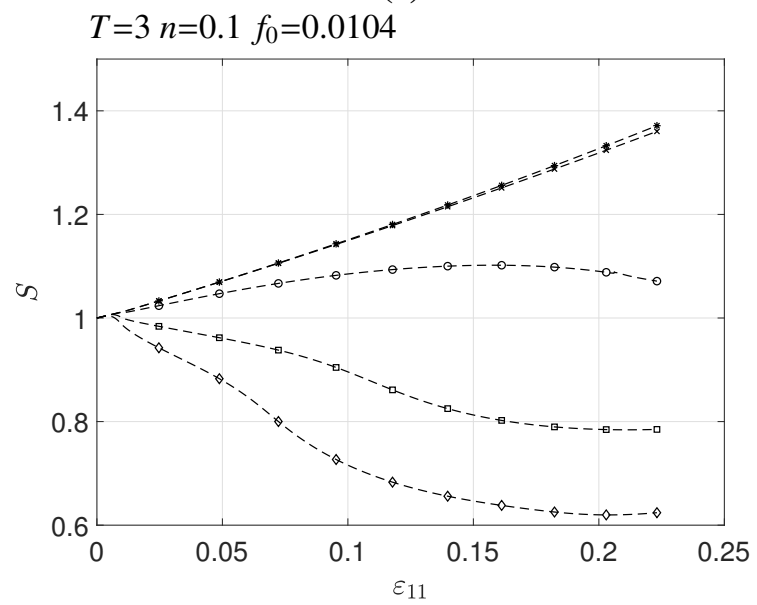

Fig. 3 Aspect ratio of the void as function of logarithmic axial strain for $f_{0}=0.0104$ and $n=0.1$ with stress triaxialites: (a) $T=1$, (b) $T=2$ and (c) $T=3$. 


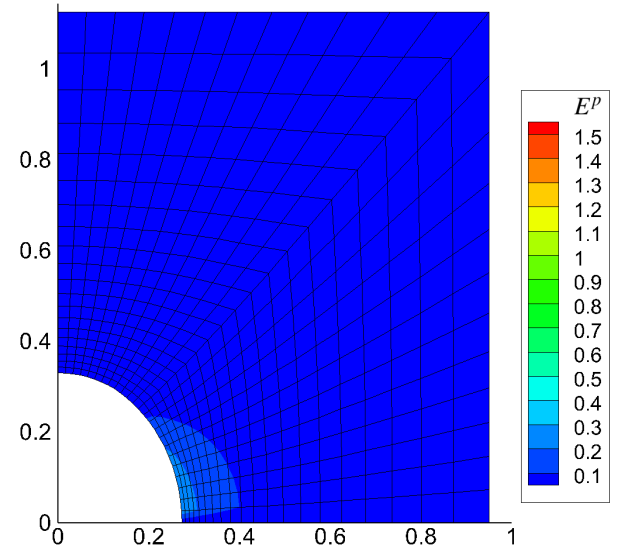

(a) $T=1, \frac{L_{D}}{R_{c}}=0$

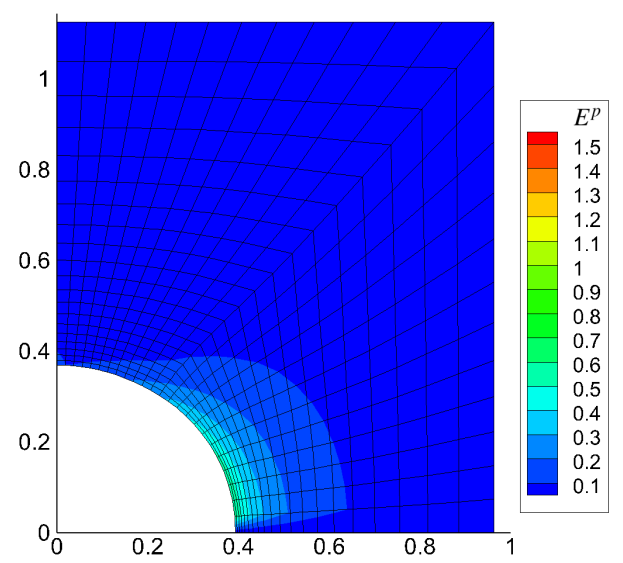

(c) $T=2, \frac{L_{D}}{R_{c}}=0$

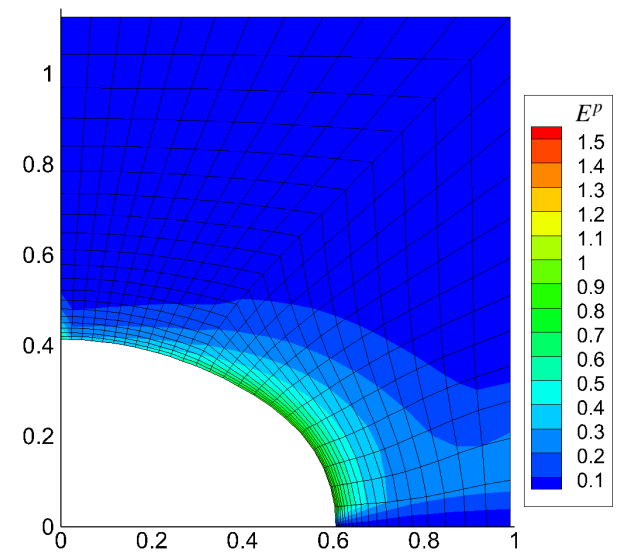

(e) $T=3, \frac{L_{D}}{R_{c}}=0$

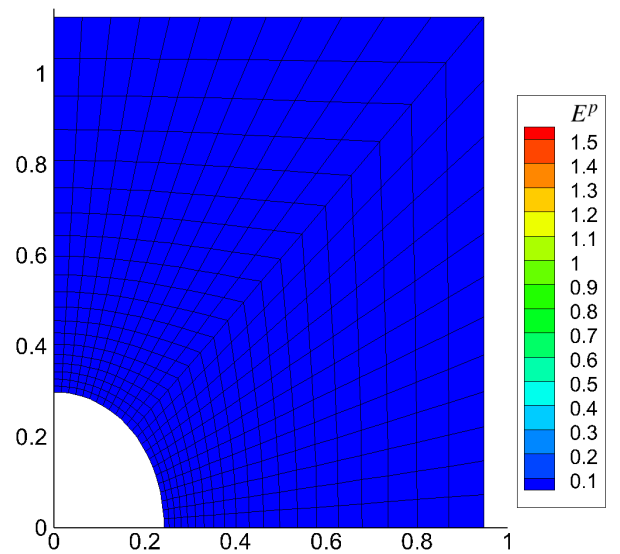

(b) $T=1, \frac{L_{D}}{R_{c}}=0.1$

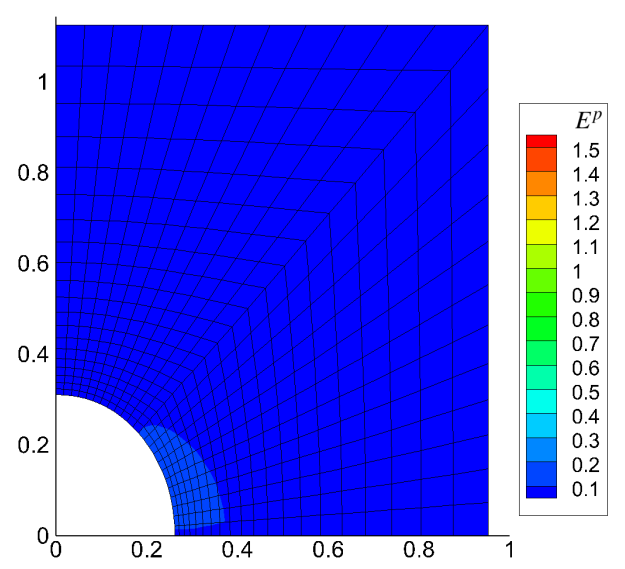

(d) $T=2, \frac{L_{D}}{R_{c}}=0.1$

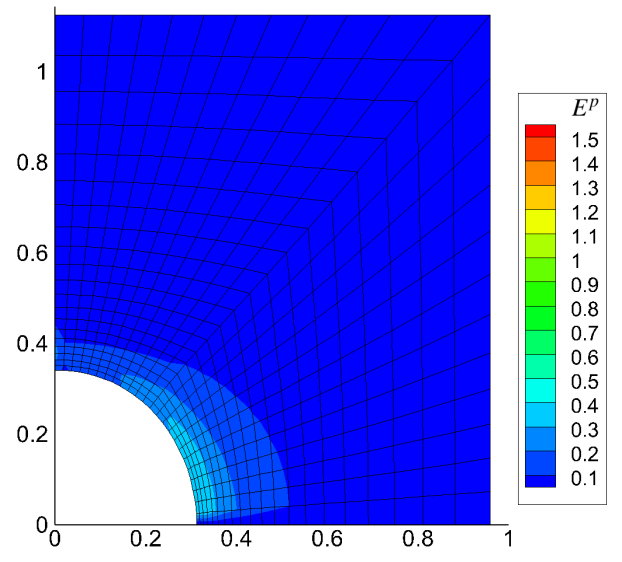

(f) $T=3, \frac{L_{D}}{R_{c}}=0.1$

Fig. 4 Deformed meshes and contours of $E^{p}$ for $f_{0}=0.0104$ and $n=0.1$ at $\varepsilon_{11}=0.118$ for three triaxialities, from top to bottom: $T=1,2$ and 3, at two measures of logarithmic axial strain. The left column shows results at $\frac{L_{D}}{R_{c}}=0$ and the right column at $\frac{L_{D}}{R_{c}}=0.1$. The 19 initial radius, $R_{0}$, is 0.25 . 

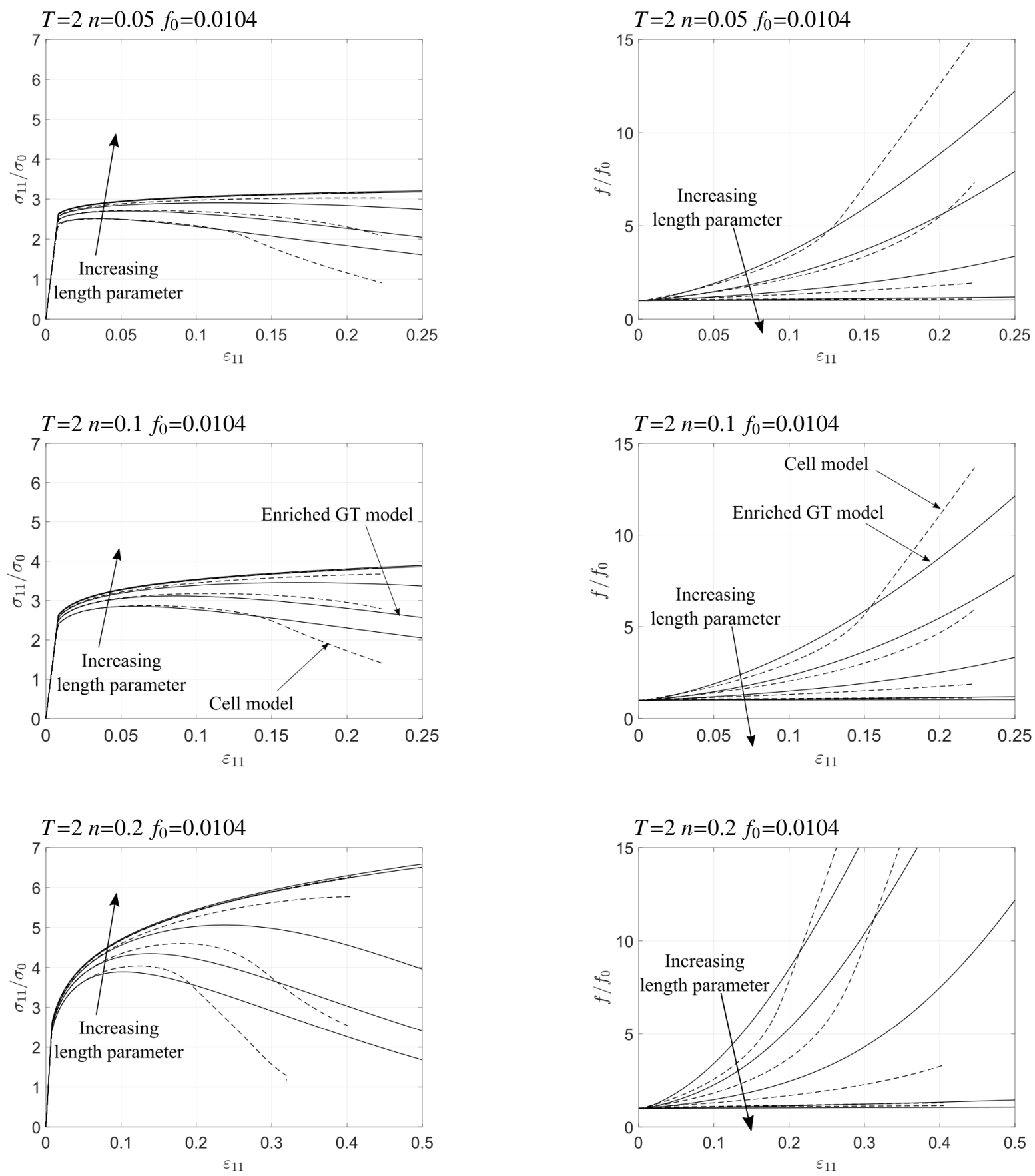

Fig. 5 Finite element results for $f_{0}=0.0104$ and $T=2$ with hardening exponents $n=0.05,0.1$ and 0.2 (from the top down). The left column shows the macroscopic true axial stress-logarithmic axial strain response. The right column shows the void volume fraction as function of logarithmic axial strain. The solid lines are the results from the enriched Gurson model, and the cell model results are displayed through the dashed lines. The five length parameters are $\frac{L_{D}}{R_{c}}=0,0.05,0.1,0.25$ and 0.5 . 
(a)

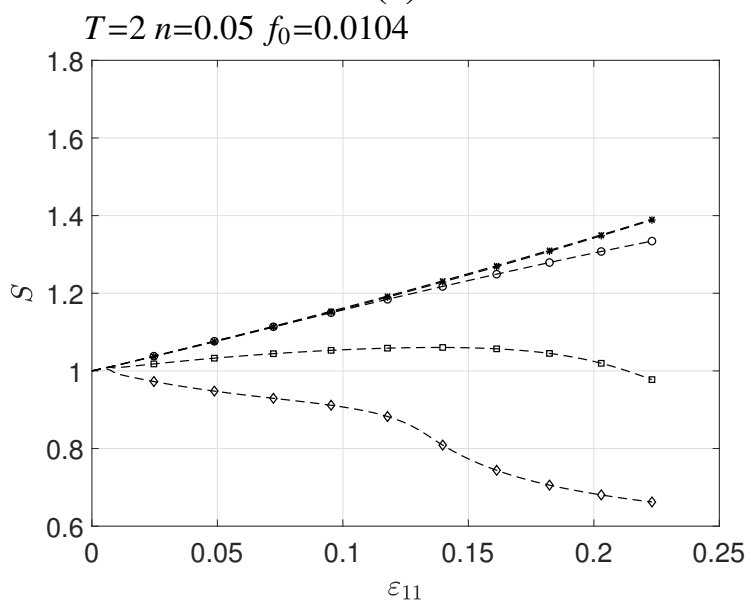

(b)

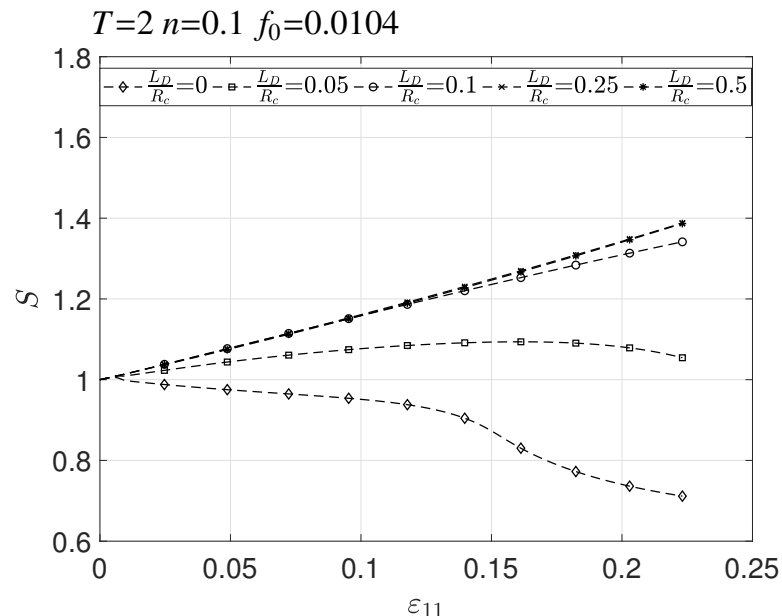

(c)

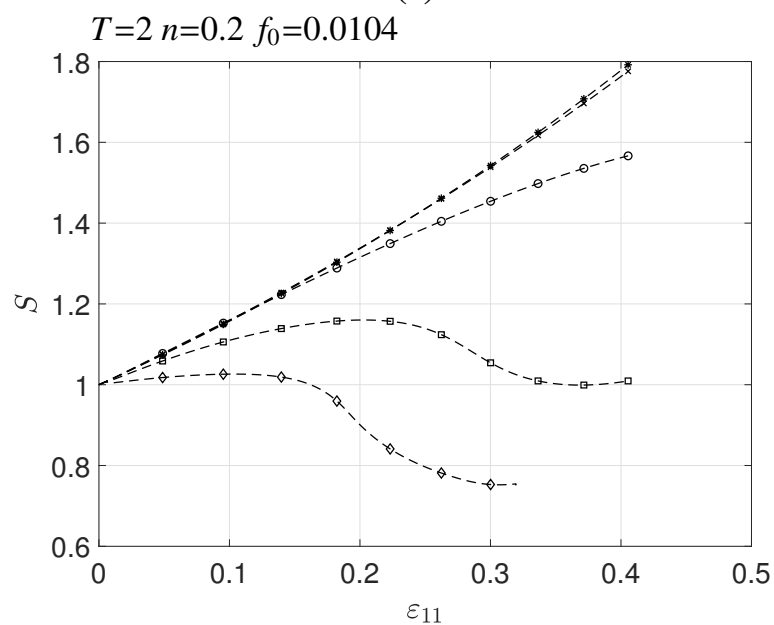

Fig. 6 Aspect ratio of the void as function of axial strain for $f_{0}=0.0104$ and $T=2$ with different hardening exponents. (a) $n=0.05$; (b) $n=0.1$; (c) $n=0.2$. 


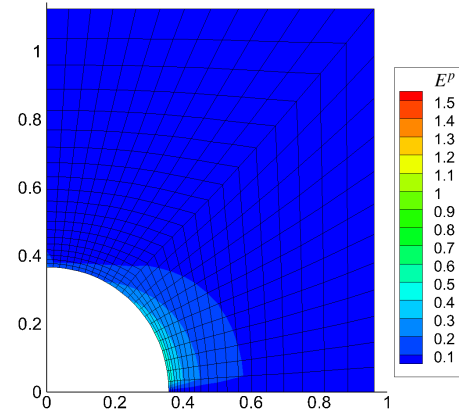

(a)

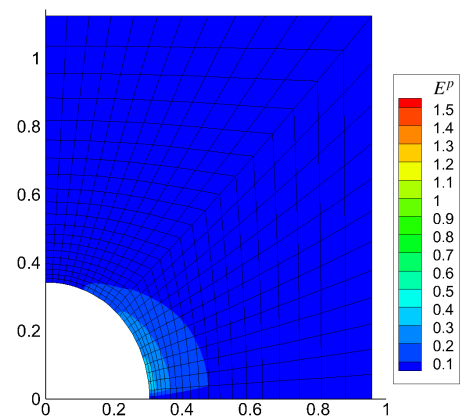

(d)

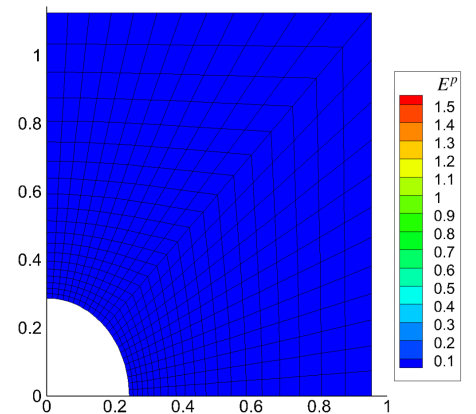

(g)

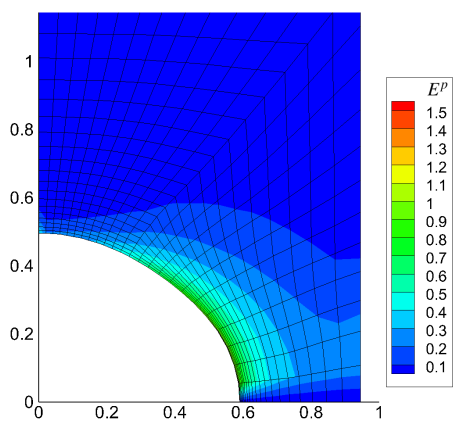

(b)

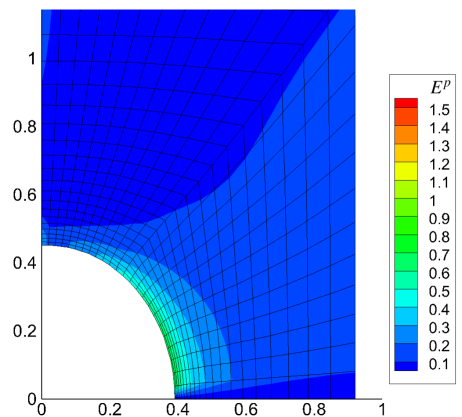

(e)

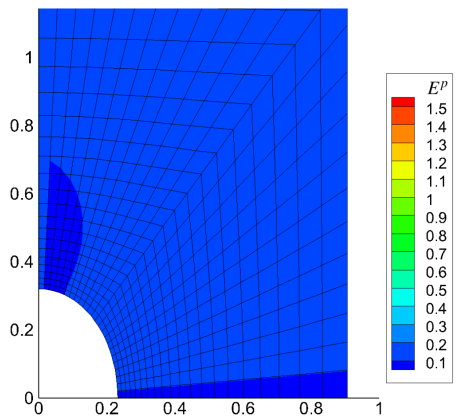

(h)

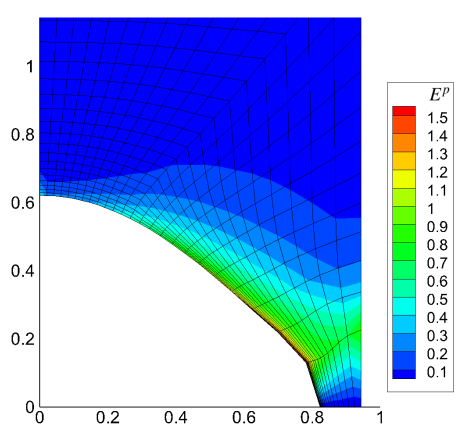

(c)

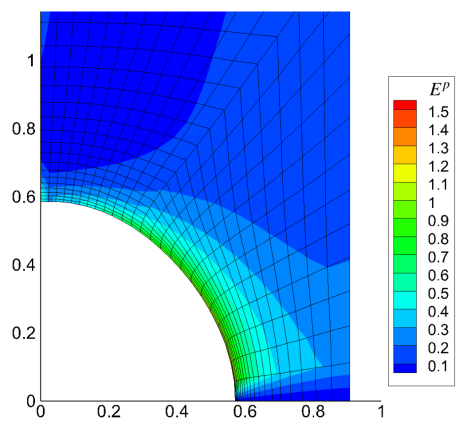

(f)

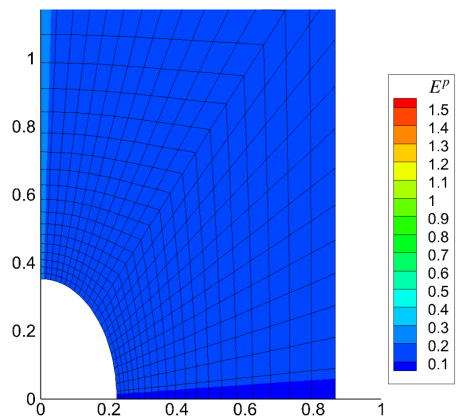

(i)

Fig. 7 Deformed meshes and contours of $E^{p}$ for $f_{0}=0.0104, T=2$ and $n=0.2$ for three different intrinsic length parameters. The top row shows results for $\frac{L_{D}}{R_{c}} \rightarrow 0$, the middle row for $\frac{L_{D}}{R_{c}}=0.05$ and the last row for $\frac{L_{D}}{R_{c}}=0.5$. The columns represent axial strain of, from left to right: $\varepsilon_{11}=0.118,0.223$ and 0.318 . 

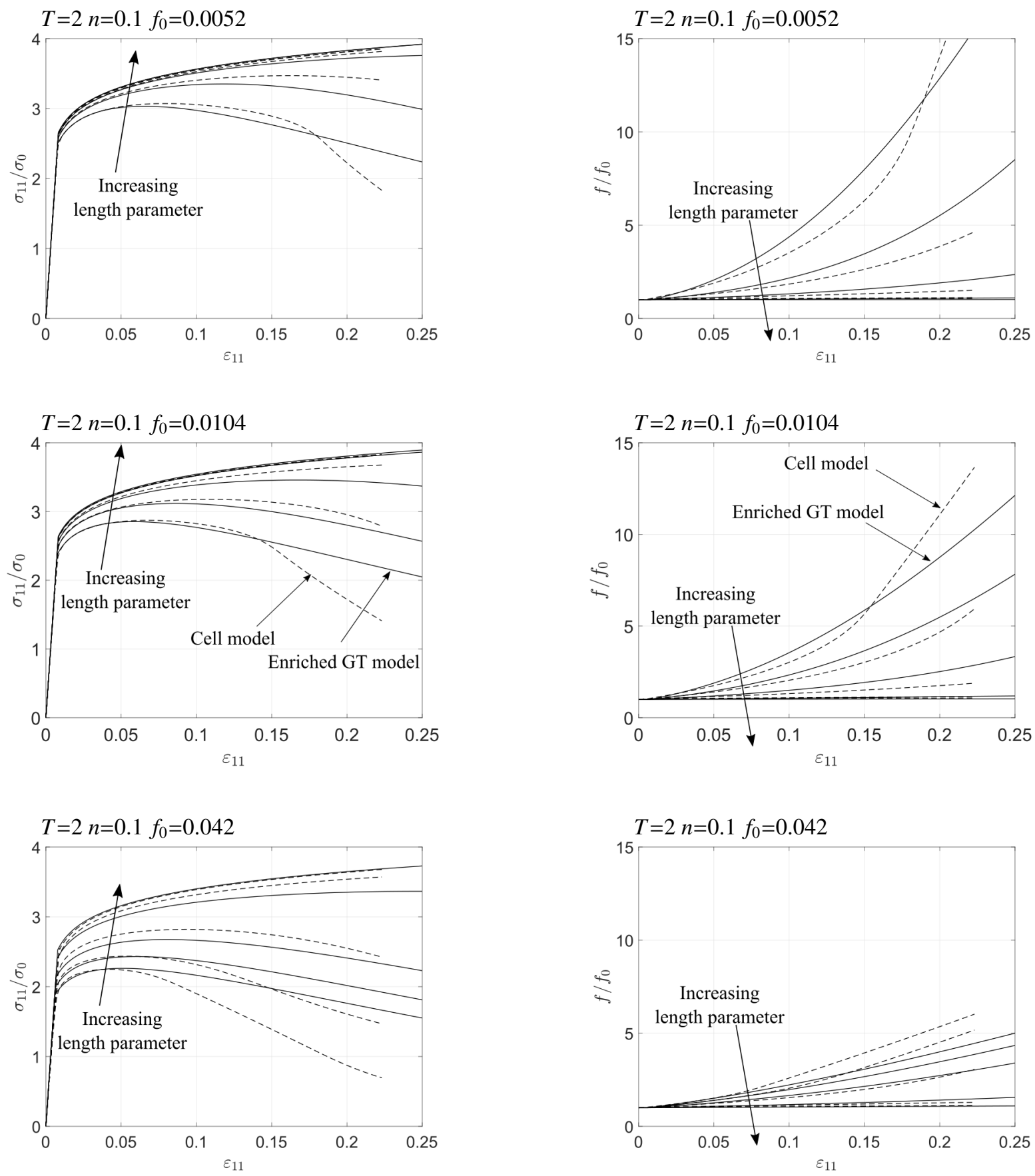

Fig. 8 Finite element results for $T=2$ and $n=0.1$ with initial void volume fraction $f_{0}=0.51 \%, 0.0104$ and 0.042 (from the top down.) The left column shows the macroscopic true axial stress-logarithmic axial strain response. The right column shows the void volume fraction as function of logarithmic axial strain. The solid lines are the results from the enriched Gurson model, and the cell model results are displayed through the dashed lines. The five length parameters are $\frac{L_{D}}{R_{c}}=0,0.05,0.1,0.25$ and 0.5 . 
(a)

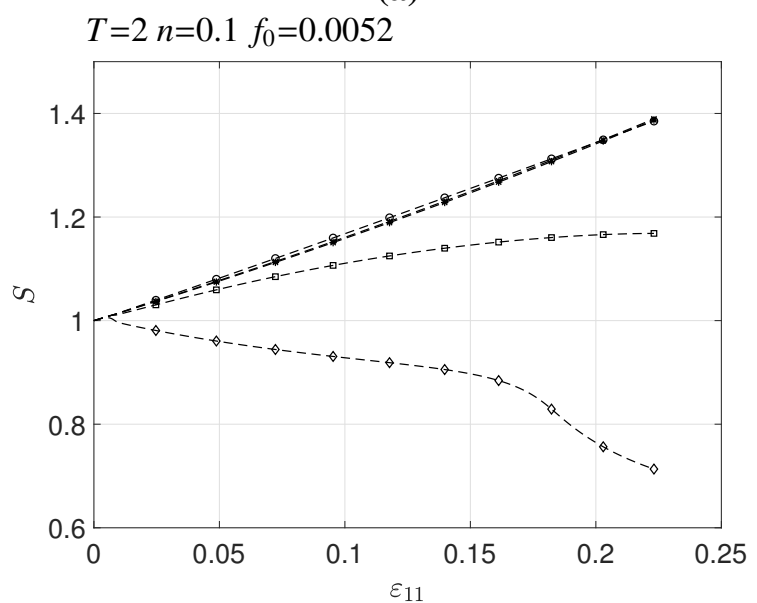

(b)

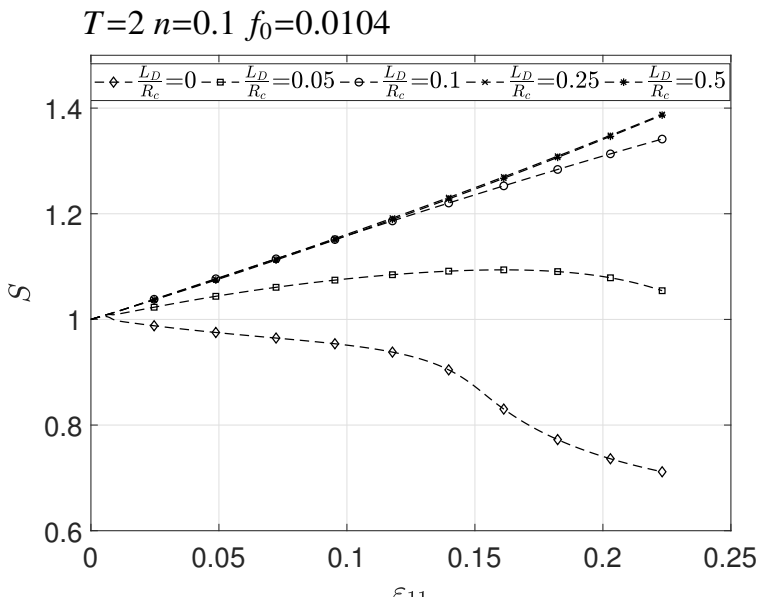

(c)

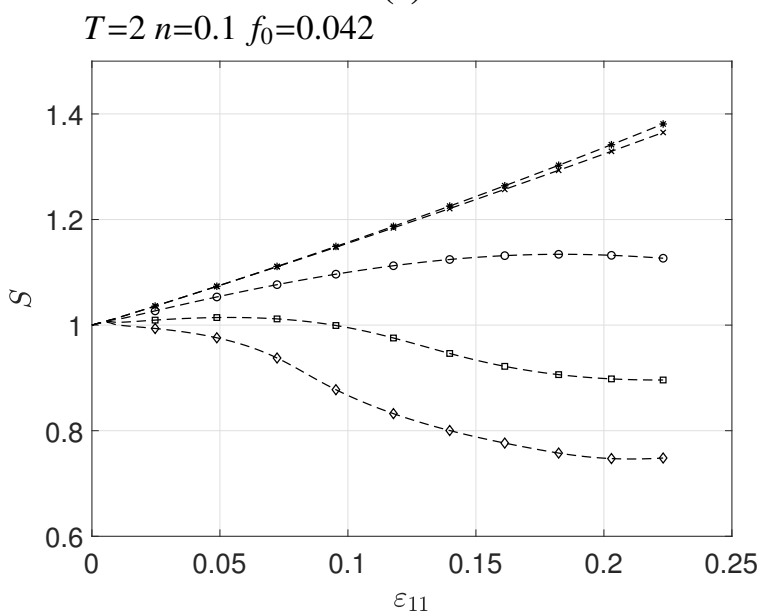

Fig. 9 Aspect ratio of void as function of axial strain for $T=2, n=0.1$ with different initial void volume fractions. (a) $f_{0}=0.0052$; (b) $f_{0}=0.0104$; (c) $f_{0}=0.042$. 


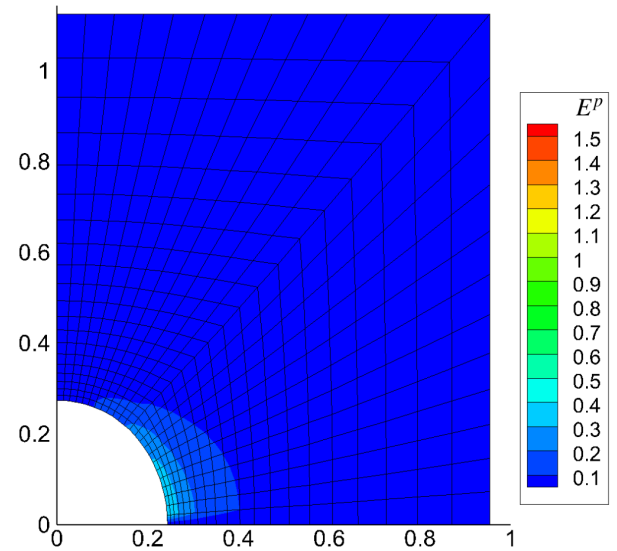

(a) $\varepsilon_{11}=0.118$

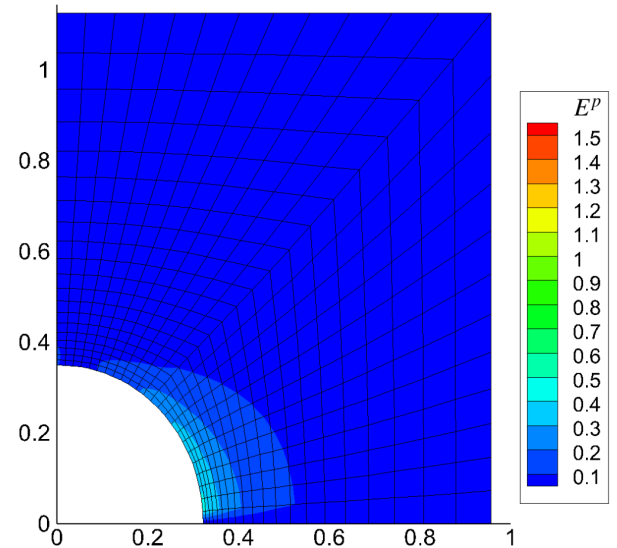

(c) $\varepsilon_{11}=0.118$

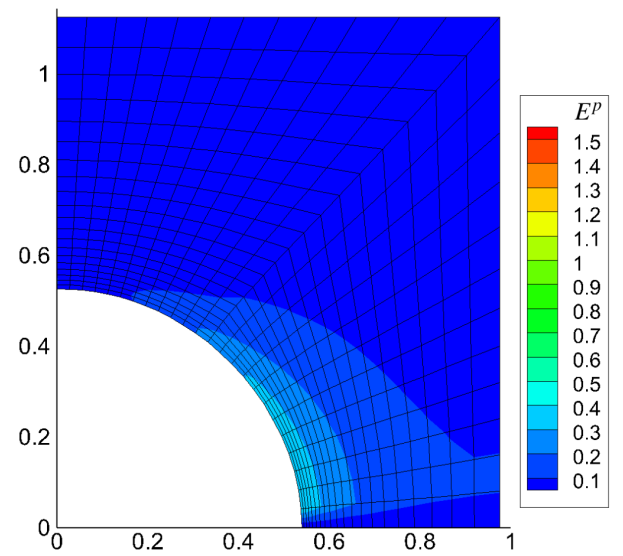

(e) $\varepsilon_{11}=0.118$

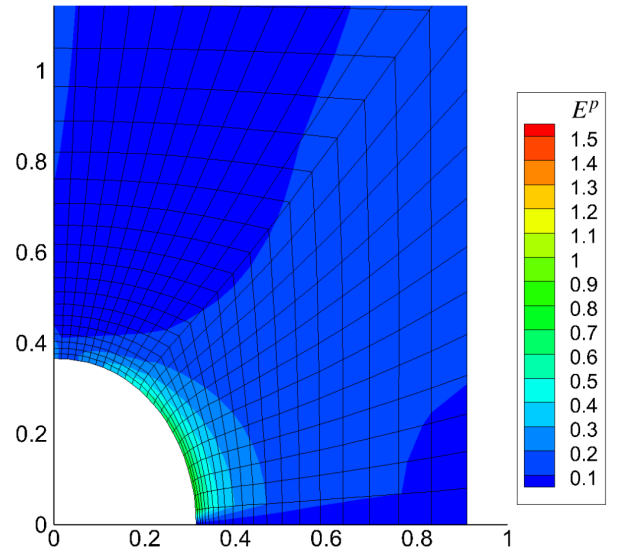

(b) $\varepsilon_{11}=0.223$

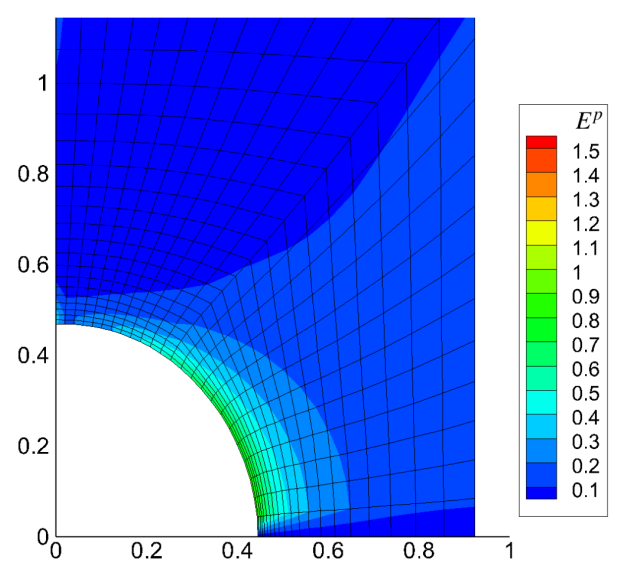

(d) $\varepsilon_{11}=0.223$

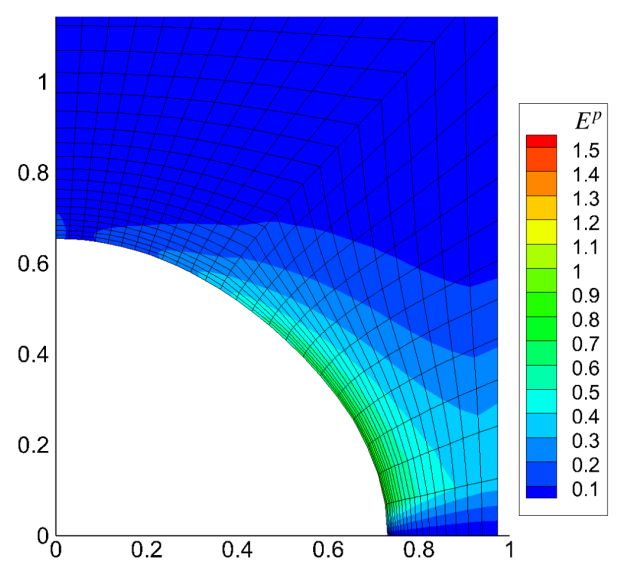

(f) $\varepsilon_{11}=0.223$

Fig. 10 Deformed meshes and contours of $E^{p}$ for $\mathrm{n}=0.1, T=2$ and $\frac{L_{D}}{R_{C}}=0.05$ for three different initial void volume fractions at two different measures of logarithmic axial strain: (a-b) $f_{0}=0.0052\left(R_{0}=0.2\right)$; (c-d) $f_{0}=0.0104\left(R_{0}=0.25\right) ;(\mathrm{e}-\mathrm{f}) f_{0}=0.042\left(R_{0}=0.4\right)$ 


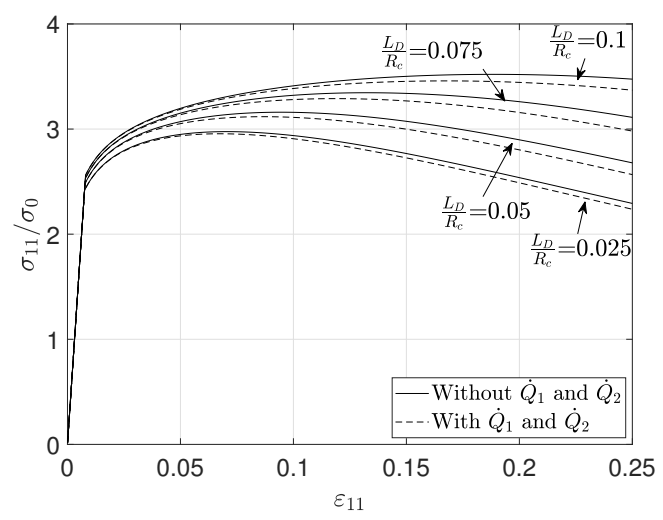

(a)

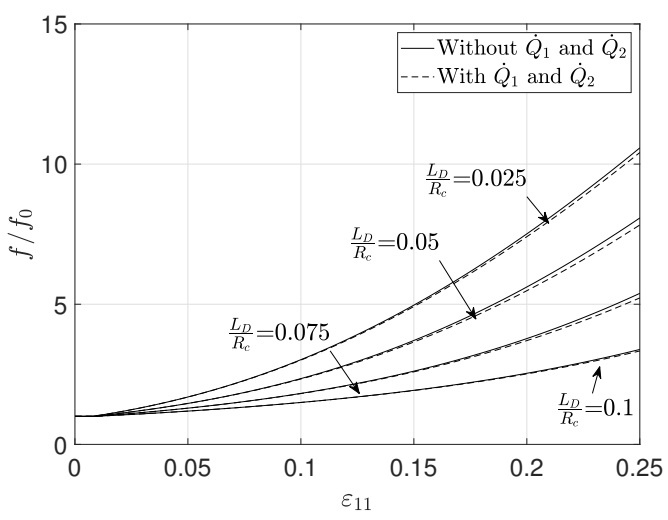

(b)

Fig. 11 Response curves (a) and void evolution (b) for four different length parameters. The solid lines are results for the enriched Gurson model without $\dot{Q}_{1}$ and $\dot{Q}_{2}$. The dashed lines show results for the enriched Gurson model with $\dot{Q}_{1}$ and $\dot{Q}_{2}$ accounted for in the consistency condition. $T=2, n=0.1$ and $f_{0}=0.0104$. 


\section{Conclusion}

The present work has investigated a gradient enriched strain hardening Gurson-Tvergaard model incorporating an intrinsic length parameter tied to the void size in a ductile material model, as proposed by Niordson and Tvergaard (2019). The length scale parameter enters the constitutive equations through prefactors of the usual $q_{1}$ and $q_{2}$ parameters. The enriched GT model was modelled as a single point representing a porous continuum. A cell model incorporating a discrete void in a strain gradient plasticity governed matrix was used as benchmark for the simulation. A parametric study of stress triaxiality, strain hardening exponent and initial void volume fraction has been carried out. Three values of all the parameters in the study have been investigated for five values of the intrinsic length parameter. The enriched Gurson model is found to capture the elevated yield point and suppressed void growth with increasing gradient hardening for all parameters investigated. The length parameter influenced the void shape evolution, which is not captured by the enriched Gurson model as the voids are accounted for solely through the damage parameter $f$, the void volume fraction. Void shape was found significant for the material response as the region between the voids perpendicular to the main straining axis is of greater importance than the void volume itself, i.e. oblate voids can withstand less axial stress than prolate voids due to the size of the intervoid ligaments. A material with sufficient gradient hardening will not be affected by such effects as the gradients inhibit void evolution and thereby localisation, overall softening and coalescence. 


\section{Acknowledgement}

This research was financially supported by Danish Council for Independent Research through the research project "Advanced Damage Models with InTrinsic Size Effects" (Grant no: DFF7017-00121).

\section{References}

[1] Ashby, M., 1970. The deformation of plastically non-homogeneous materials. Philosophical Magazine 21 (170), 399-424. doi: $10.1080 / 14786437008238426$

[2] Bishop, J., Hill, R., 1951. A theory of the plastic distortion of a polycrystalline aggregate under combined stresses. The London, Edinburgh, and Dublin Philosophical Magazine and Journal of Science 42 (327), 414427.

doi: $10.1080 / 14786445108561065$

[3] Budiansky, B., Hutchinson, J., Slutsky, S., 1982. Void growth and collapse in viscous solids. In: Mechanics of Solids: The Rodney Hill 60th Anniversary Volume. Elsevier, pp. 13-45.

doi: 10.1016/B978-0-08-025443-2.50009-4

[4] Dormieux, L., Kondo, D., 2010. An extension of gurson model incorporating interface stresses effects. International Journal of Engineering Science 48 (6), 575-581. doi: $10.1016 / j$.ijengsci.2010.01.004

[5] Fleck, N., Muller, G., Ashby, M., Hutchinson, J., 1994. Strain gradient plasticity: theory and experiment. Acta Metallurgica et Materialia 42 (2), 475-487.

doi: 10.1016/0956-7151 (94)90502-9

[6] Fleck, N. A., Hutchinson, J. W., 1993. A phenomenological theory for strain gradient effects in plasticity. Journal of the Mechanics and Physics of Solids 41 (12), 1825-1857. doi: 10 .1016/0022-5096 (93) 90072-N

[7] Fleck, N. A., Hutchinson, J. W., 1997. Strain gradient plasticity. Advances in Applied Mechanics 33, $296-361$.

[8] Fleck, N. A., Hutchinson, J. W., 2001. A reformulation of strain gradient plasticity. Journal of the Mechanics and Physics of Solids 49 (10), 2245-2271. doi: $10.1016 / \mathrm{S} 0022-5096(01) 00049-7$

[9] Fleck, N. A., Willis, J. R., 2009. A mathematical basis for strain-gradient plasticity theory: Part II: tensorial plastic multiplier. Journal of the Mechanics and Physics of Solids 57 (7), 1045-1057. doi: $10.1016 / j \cdot j m p s .2009 .03 .007$

[10] Gao, H., Huang, Y., 2001. Taylor-based nonlocal theory of plasticity. International Journal of Solids and Structures 38 (15), 2615-2637. doi: $10.1016 / \mathrm{S} 0020-7683(00) 00173-6$

[11] Gudmundson, P., 2004. A unified treatment of strain gradient plasticity. Journal of the Mechanics and Physics of Solids 52 (6), 1379-1406. doi: $10.1016 / j \cdot j m p s .2003 .11 .002$

[12] Gurson, A. L., 1977. Continuum theory of ductile rupture by void nucleation and growth. part I: yield criteria and flow rules for porous ductile media. Journal of Engineering Materials and Technology 99 (1), 2-15. doi: $10.1115 / 1.3443401$

[13] Hutchinson, J. W., 2000. Plasticity at the micron scale. International Journal of Solids and Structures 37 (1-2), 225-238. doi: $10.1016 / \mathrm{S} 0020-7683(99) 00090-6$

[14] Koplik, J., Needleman, A., 1988. Void growth and coalescence in porous plastic solids. International Journal of Solids and Structures $24(8), 835-853$. doi: $10.1016 / 0020-7683$ (88) 90051-0 
[15] Ma, Q., Clarke, D. R., 1995. Size dependent hardness of silver single crystals. Journal of Materials Research $10(4), 853-863$. doi: $10.1557 /$ JMR. 1995.0853

[16] McMeeking, R., Hom, C., 1990. Finite element analysis of void growth in elastic-plastic materials. International Journal of Fracture 42 (1), 1-19. doi: $10.1007 / \mathrm{BF} 00018610$

[17] Monchiet, V., Bonnet, G., 2013. A gurson-type model accounting for void size effects. International Journal of Solids and Structures 50 (2), 320-327. doi: $10.1016 /$ j.ijsolstr.2012.09.005

[18] Monchiet, V., Kondo, D., 2013. Combined voids size and shape effects on the macroscopic criterion of ductile nanoporous materials. International Journal of Plasticity 43, 20-41. doi: $10.1016 / j$.ijplas.2012.10.007

[19] Nielsen, K. L., Niordson, C. F., 2013. A 2d finite element implementation of the fleck-willis strain-gradient flow theory. European Journal of Mechanics-A/Solids 41, 134-142. doi: $10.1016 / \mathrm{j}$. euromechsol.2013.03.002

[20] Nielsen, K. L., Niordson, C. F., 2014. A numerical basis for strain-gradient plasticity theory: rate-independent and rate-dependent formulations. Journal of the Mechanics and Physics of Solids 63, 113-127. doi: $10.1016 / j \cdot j m p s .2013 .09 .018$

[21] Niordson, C. F., Tvergaard, V., 2019. A homogenized model for size-effects in porous metals. Journal of the Mechanics and Physics of Solids 123, 222-233. doi: $10.1016 / j \cdot j m p s .2018 .09 .004$

[22] Stelmashenko, N., Walls, M., Brown, L., Milman, Y. V., 1993. Microindentations on W and Mo oriented single crystals: an STM study. Acta Metallurgica et Materialia 41 (10), 2855-2865. doi: $10.1016 / 0956-7151(93) 90100-7$

[23] Stölken, J. S., Evans, A., 1998. A microbend test method for measuring the plasticity length scale. Acta Materialia 46 (14), 5109-5115. doi: $10.1016 /$ S1359-6454(98) 00153-0

[24] Tvergaard, V., 1981. Influence of voids on shear band instabilities under plane strain conditions. International Journal of Fracture 17 (4), 389-407. doi: $10.1007 / \mathrm{BF} 00036191$

[25] Tvergaard, V., 1982. Ductile fracture by cavity nucleation between larger voids. Journal of the Mechanics and Physics of Solids 30 (4), 265-286. doi: $10.1016 / 0022-5096(82) 90033-3$

[26] Wen, J., Huang, Y., Hwang, K., Liu, C., Li, M., 2005. The modified gurson model accounting for the void size effect. International Journal of Plasticity 21 (2), 381-395. doi: $10.1016 /$ j.ijplas.2004.01.004 